\title{
A Multi-Sensor Data Fusion Method by Combining Near-neighbor Method and Fuzzy Inference
}

\author{
Jianxing Lianga, Yanting Wang ${ }^{b}$, Hong Yin ${ }^{c}$ and Guolin Liu \\ China Luoyang Electronic Equipment Testinh Center, Luoyang 471003, China \\ ajianxingliang@hotmail.com, bWangyanting454799@163.com, cxfdsj26@sohu.com
}

Keywords: target tracking, accuracy, fuzzy inference, near-neighbor method.

\begin{abstract}
Aiming at the practical issue of multi-sensor data fusion in target tracking, this paper proved that the accuracy of multi-sensor data fusion is better than that of any one sensor in terms of accuracy definition. According to the proof method, we proposed a multi-sensor data fusion method by combinating the near-neighbor method and fuzzy inference. The method combines the near-neighbor method and weighted average of fusion inference method in engineering practice. This paper theoretically proved that, as the number of sensors increases, the accuracy of data fusion is higher rather than worse. Numerical examples show that, our method in this paper has better results than the only weighted average fusion method of fuzzy inference.
\end{abstract}

\section{Introduction}

Recently, multi-sensor data fusion has become a very hot research field. It is a high-level key technology ${ }^{[1]}$ commonly concerned by multi-disciplinary, multi-sector and multi-domain. In the multi-sensor data fusion system, it is necessary to synthesize the same target track data from multi-sensor. This can obtain higher accuracy. Due to the different position of each sensor in the process of data fusion, different fusion methods, such as the nearest neighbor method, the fusion method based on weights, the fusion method based on fuzzy inference and the fusion method based on neural network are generated ${ }^{[2]}$. It is proved in theory that higher accuracy of data fusion will be obtained from more sensors. But this conclusion is still questioned by many people. Because it is only proved in data variance, which is in data precision aspect.

The definition of data accuracy is relatively confusing at present. It is generally believed that data precision is the accuracy of data. However, data precision is difficult to characterize the proximity of measured value to its true value. So the accuracy of data refers to the degree of agreement between the measured values and the proximity to their true values, which is a comprehensive concept. So accuracy is used to describe the effect of data fusion in target track.

Based on proof of data precision in paper [1 2], it is proved that the accuracy of fusion data from multi-sensor is better than all measurement accuracy. Then, a multi-sensor data fusion method by combinating the near-neighbor method and fuzzy inference is proposed, which combines the near-neighbor method and weighted average of fusion inference method in engineering practice.

\section{Fuzzy reasoning method of multi-sensor fusion}

In the target tracking system, uncertainty is present in the whole tracking process due to the complexity of tracking environment, the uncertrain num of targets and the mutability of moving objects. Which is called ambiguity. Fuzzy reasoning can carry out logical reasoning ${ }^{[5]}$ when the connotation and extension of concepts are not clear or the relation between concepts is broken. Therefore, fuzzy reasoning is used in target data fusion.

The measurement error distance and the variance of the last 5 frames of measurement error is used to reflect the accuracy and precision of the sensor measurements. They are inputs to the fuzzy inference system in this paper, represented by $e(n)$ and $\sigma^{2}(n)$ respectively. 


$$
\begin{aligned}
& e(n)=\sqrt{\vec{e}^{\prime}(n) \vec{e}(n)}, \vec{e}(n)=Z(n)-H(n) \hat{X}(n) \\
& \sigma^{2}(n)=\frac{1}{5} \sum_{k=0}^{4}[e(n-k)-\mu(n)]^{2}, \mu(n)=\operatorname{median}[e(n), \cdots, e(n-4)]
\end{aligned}
$$

$\mu(n)$ is the median estimate of the error distance for the last 5 frames. In this paper, the median estimate is used because it is more robust than the mean value, this can effectively avoid the influence of outliers. Fuzzy variables $E(n)$ and $V(n)$ respectively represent the fuzzy values of the measurement error distance $e(n)$ and the variance of the last 5 frames of measurement error $\sigma^{2}(n)$. The fuzzy set theory domains for the fuzzy variables $E(n)$ and $V(n)$ are $\{V S, S, M, B, V B\}$ and $\{V S, S, M, B, V B\}$. The membership functions of each fuzzy set are shown in Fig 1 and Fig 2 .

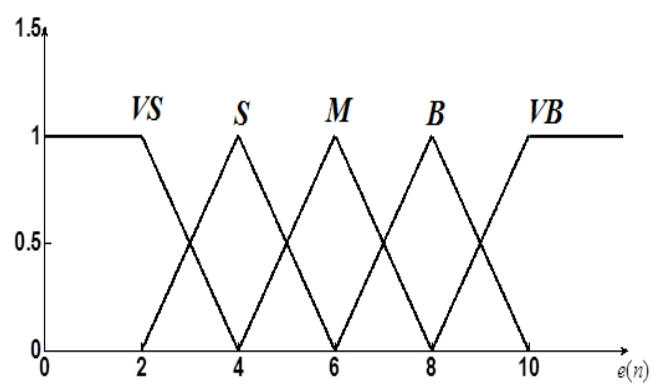

Fig. 1 membership function of fuzzy set $E(n)$

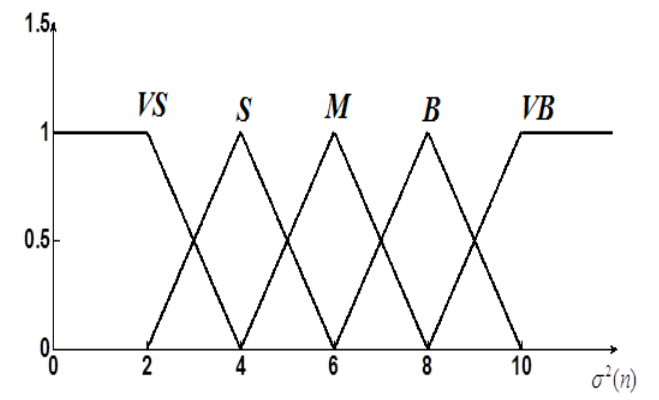

Fig. 2 membership function of fuzzy set $V(n)$

The Takagi-Sugeno fuzzy system is adopted in this paper because of its unnecessary bluring ${ }^{[7 \sim 8]}$. Suppose the fuzzy variable $P(n)$ is the sensor satisfaction degree of the current frame time, and its fuzzy set theory domain is $\{V L, L, M, H, V H\}$. The fuzzy satisfaction of $\{V L, L, M, H, V H\}$ is expressed by $g_{1}, g_{2}, \cdots, g_{5}$, then we can assume: $g_{1}=0.99, g_{2}=0.8, g_{3}=0.5, g_{4}=0.2, g_{5}=0.01$.

The fuzzy inference principle used in this paper is that the smaller the error $e(n)$ is, the higher the sensor accuracy at the current frame time is, and the smaller the variance $\sigma^{2}(n)$ is, the higher the sensor accuracy at the current frame time is. The inference rules adopts the form of IF-THEN. For example, IF $E(n)$ is $V S$, THEN the fuzzy satisfaction degree is $E_{V S}(n) g_{1}, E_{V S}(n)$ represents the ambiguity size corresponding to the measurement error $e(n)$ when $E(n)$ is $V S$. IF $V(n)$ is $S$, THEN the fuzzy satisfaction degree is $V_{S}(n) g_{2}, V_{S}(n)$ represents the ambiguity size corresponding to the variance $\sigma^{2}(n)$ of the last 5 frames measurement error $e(n)$ when $E(n)$ is $S$.

The Max-Min inference mechanism, or the arithmetic average operator can be used to determine the final satisfaction degree.

Suppose a target is measured by m sensors. After the satisfaction of each sensor $P_{i}(n), i=1,2, \cdots, m$ is obtained, we can get the multi-sensor fusion value of the target state by weighted average method of each sensor measurement. The specific formula is as follows: 


$$
x_{r}(n)=\sum_{i=1}^{m} x_{i} \omega_{i}(n)
$$

The weight value is $\omega_{i}(n)=P_{i}(n) / \sum_{j=1}^{m} P_{j}(n), x_{i}$ is the data from the $\mathrm{i}$ sensor.

\section{Proof: the accuracy of fusion data is better than that of any sensor measurement.}

First, it is proved that the accuracy of two sensor data fusion is better than that of any sensor.

Since the actual measured values may contain outliers, they are filtered values here. Suppose a target in three-dimensional coordinates, the first sensor measured value is $\left(x_{1}, y_{1}, z_{1}\right)$, the second sensor measured value is $\left(x_{2}, y_{2}, z_{2}\right)$, the true value of the target is $\left(x_{0}, y_{0}, z_{0}\right)$. Then the fusion value are: $x_{r}=\sum_{i=1}^{2} x_{i} \omega_{i}, y_{r}=\sum_{i=1}^{2} y_{i} \omega_{i}, z_{r}=\sum_{i=1}^{2} z_{i} \omega_{i}$.

When the measured values of the two sensors are not less than the true value, we assume $x_{1} \geq x_{2} \geq x_{0}$, then $x_{1} \geq x_{r} \geq x_{2}, x_{1}-x_{0} \geq x_{r}-x_{0} \geq x_{2}-x_{0} \geq 0$, so the accuracy of the fusion value is between the accuracy of two sensors. In order to optimize the precision of fusion value, let $x_{r}=\min \left(x_{1}, x_{2}\right)$, we can get $\left|x_{r}-x_{0}\right| \leq\left|x_{1}-x_{0}\right|,\left|x_{r}-x_{0}\right| \leq\left|x_{2}-x_{0}\right|$.

When the measured values of the two sensors are less than the true value, we assume $x_{1} \leq x_{2} \leq x_{0}$, then $x_{1} \leq x_{r} \leq x_{2}, x_{1}-x_{0} \leq x_{r}-x_{0} \leq x_{2}-x_{0} \leq 0$, so the accuracy of the fusion value is between the accuracy of two sensors. In order to optimize the precision of fusion value, let $x_{r}=\max \left(x_{1}, x_{2}\right)$, we can get $\left|x_{r}-x_{0}\right| \leq\left|x_{1}-x_{0}\right|,\left|x_{r}-x_{0}\right| \leq\left|x_{2}-x_{0}\right|$.

When the true value is between the measured values of the two sensors, we assume $x_{1} \geq x_{0} \geq x_{2}$, then $\quad x_{1} \geq x_{r} \geq x_{2} \quad, \quad x_{r}-x_{0}=\omega_{1}\left(x_{1}-x_{0}\right)+\omega_{2}\left(x_{2}-x_{0}\right) \quad$. If $\quad\left|x_{1}-x_{0}\right| \leq\left|x_{2}-x_{0}\right| \quad, \quad$ then

$$
\begin{aligned}
\left|x_{r}-x_{0}\right|-\left|x_{1}-x_{0}\right| & =\left|\omega_{1}\left(x_{1}-x_{0}\right)+\omega_{2}\left(x_{2}-x_{0}\right)\right|-\left|x_{1}-x_{0}\right| . \\
\text { If }\left|\omega_{1}\left(x_{1}-x_{0}\right)\right| & \geq\left|\omega_{2}\left(x_{2}-x_{0}\right)\right| \text {, then }\left|x_{r}-x_{0}\right|-\left|x_{1}-x_{0}\right|=\omega_{2}\left(x_{2}-x_{1}\right) \leq 0 . \\
\text { If }\left|\omega_{1}\left(x_{1}-x_{0}\right)\right| & \leq\left|\omega_{2}\left(x_{2}-x_{0}\right)\right| \text {, then }\left|x_{r}-x_{0}\right|-\left|x_{1}-x_{0}\right|=-\left(\omega_{1}+1\right)\left(x_{1}-x_{0}\right)-\omega_{2}\left(x_{2}-x_{0}\right) .
\end{aligned}
$$

The fuzzy inference principle is the smaller the error, the higher the weight of the sensor at the moment of the current frame. So $\omega_{1} \geq \omega_{2}, \omega_{1}+1 \geq 3 \omega_{2}$, and $\left|x_{1}-x_{0}\right|$ is smaller than $\left|x_{2}-x_{0}\right|$. The bigger $\omega_{1}$ is, the higher probability we get $\left|x_{r}-x_{0}\right|-\left|x_{1}-x_{0}\right| \leq 0$ using fuzzy inference principle. Thus, when the true value is between the measured values of the two sensors, we get $\left|x_{r}-x_{0}\right| \leq\left|x_{1}-x_{0}\right|$, $\left|x_{r}-x_{0}\right| \leq\left|x_{2}-x_{0}\right|$.

In conclusion, we can get $\left|x_{r}-x_{0}\right| \leq\left|x_{1}-x_{0}\right|,\left|x_{r}-x_{0}\right| \leq\left|x_{2}-x_{0}\right|$ in any condition. This proved that the accuracy of the fusion value $x_{r}$ is better than that of $x_{1}, x_{2}$. Similarly the accuracy of the fusion value $y_{r}$ is better than that of $y_{1}, y_{2}$, the accuracy of the fusion value $z_{r}$ is better than that of $z_{1}, z_{2}$. So the accuracy of two sensor data fusion is better than that of any sensor. When there are three sensors, first we get the fusion value from two sensors, its accuracy is better than both the sensors. Then the fusion value is fused with the third measured value. Similarly the accuracy of the fusion value is better than any of the three sensors. And so on, the conclusion is get that the accuracy of multi-sensor data fusion is better than that of any sensor. Due to the proof that the precision of multi-sensor data fusion is better than that of any sensor, the conclusion is proved that the accuracy of multi-sensor data fusion is better than that of any sensor. 


\section{A Multi-Sensor Data Fusion Method by Combinating Near-neighbor Method and Fuzzy Inference}

After using the current statistical model to get $X(k+1 \mid k+1), P_{i}(k+1 \mid k+1), i=1,2, \cdots, m$, we can fuse with fuzzy reasoning to get: $X_{r}(k+1 \mid k+1)=\sum_{i=1}^{m} X_{i}(k+1 \mid k+1) W_{i}(n)$, $P_{r}(k+1 \mid k+1)=\sum_{i=1}^{m} P_{i}(k+1 \mid k+1) W_{i}(n)$. Then we bring $X_{r}(k+1 \mid k+1)$ and $P_{r}(k+1 \mid k+1)$ into Kaman filter. This combines Kaman filter and fuzzy inference.

According to the above proving process, first the combination of Kaman filter and fuzzy inference is completed. Then, if the truth value is between the filtered values of each sensor, the weights are determined by fuzzy inference for data fusion, if the filtered value of each sensor is not less than or not greater than the true value, the nearest neighbor method is used to fuse the data. This combinates the nearest neighbor method and fuzzy reasoning.

If you do so in the project, you need to find the true value. But the true value can never be get, it can only be replaced by a prediction value that is close to it. In practice, the fusion value of fuzzy reasoning $X_{r}(k+1 \mid k+1)$ can be chosen as true value, but it can not be compared with the filtered value $X_{i}(k+1 \mid k+1)$ and $X_{r}(k+1 \mid k+1)$. Because $X_{i}(k+1 \mid k+1)$ and $X_{r}(k+1 \mid k+1)$ is related, $X_{r}(k+1 \mid k+1)$ is always among $X_{i}(k+1 \mid k+1)$. To solve this problem, the reasoning is as follows:

$$
X_{i}(k+1 \mid k+1)=\left[I-K_{i}(k+1) H(k+1)\right] X_{i}(k+1 \mid k)+K_{i}(k+1) Z_{i}(k+1)
$$

Due to $X_{i}(k+1 \mid k)=\Phi(k+1, k) X_{r}(k \mid k)+U(k) \bar{a}$, all $X_{i}(k+1 \mid k)$ are equal. $X_{r}(k+1 \mid k+1)$ and $X_{i}(k+1 \mid k)$ are the state prediction of $X(k+1 \mid k+1)$, so $X_{r}(k+1 \mid k+1)-X_{i}(k+1 \mid k) \rightarrow 0$, we get :

$$
\begin{aligned}
& X_{i}(k+1 \mid k+1)-X_{r}(k+1 \mid k+1) \\
& =\left[I-K_{i}(k+1) H(k+1)\right]\left[X_{i}(k+1 \mid k)-X_{r}(k+1 \mid k+1)\right]+K_{i}(k+1)\left[Z_{i}(k+1)-H(k+1) X_{r}(k+1 \mid k)\right] \\
& \approx K_{i}(k+1)\left[Z_{i}(k+1)-H(k+1) X_{r}(k+1 \mid k)\right]
\end{aligned}
$$

The correlation of $Z_{i}(k+1)$ and $H(k+1) X_{r}(k+1 \mid k)$ is not strong, so $X_{i}(k+1 \mid k+1)-X_{r}(k+1 \mid k+1)$ can be replaced by $Z_{i}(k+1)-H(k+1) X_{r}(k+1 \mid k)$.

\section{Example analysis}

In order to verify the effect of data fusion, this paper selects that A radar and B radar track the aircraft at same time. the radar sampling rate is $20 \mathrm{~Hz}, 5432$ points are collected, and the corresponding GPS data is the true value. We select y coordinate direction to verify conveniently. Figure 3 is the final result using the motor frequency function established with the adjacent measurement distance of the first difference, the nearest 5 frames measure error distance as the median estimate, multi-sensor data fusion method by combinating near-neighbor method and fuzzy inference. The blue line is the difference between the measured data of A radar and GPS data, the green line is the difference between the measured data of B radar and GPS data, the red line is the difference between the fusion data of two radars and GPS data. 


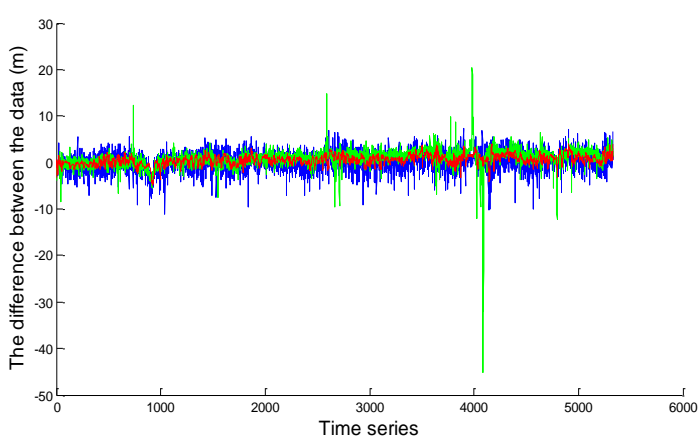

Fig. 3 the final result

1) The median estimate of the nearest 5 frames measure error distance is compared with mean value. Figure 4 is the result of substituting the median estimate of the nearest 5 frames measure error distance with the mean value in the figure 3 . It can be seen that the median estimate is more robust than the mean value, and can effectively avoid the influence of outliers.

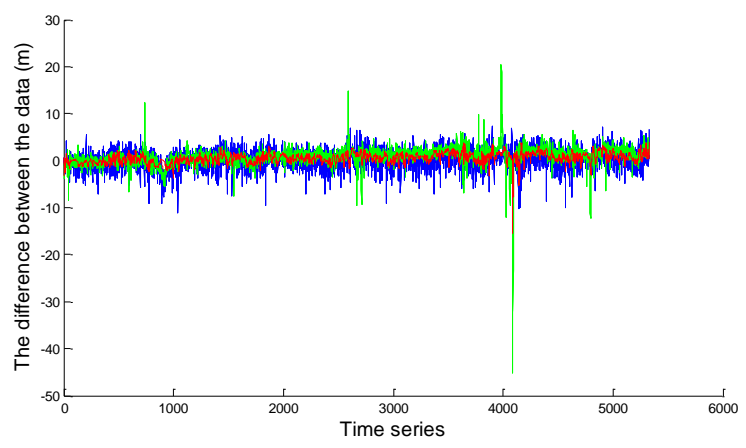

Fig. 4 the result of mean value

2) The multi-sensor data fusion method by combinating nearest neighbor method and fuzzy inference is compared with the multi-sensor data fusion method only using fuzzy inference. Compared with figure 3, figure 5 changes the multi-sensor data fusion method by combinating nearest neighbor method and fuzzy inference with the multi-sensor data fusion method only using fuzzy inference. As can be seen in Figure 3 the result is better, but not obvious. Subtracting the results of the two methods is shown in figure 6. It can be seen that the multi-sensor data fusion method by combinating nearest neighbor method and fuzzy inference improves the multi-sensor data fusion method only using fuzzy inference, and the result is better.

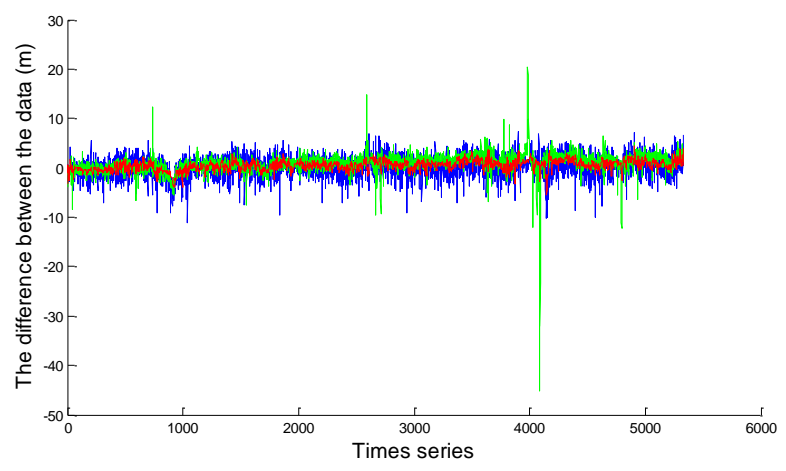

Fig. 5 the result of the multi-sensor data fusion method using fuzzy inference 


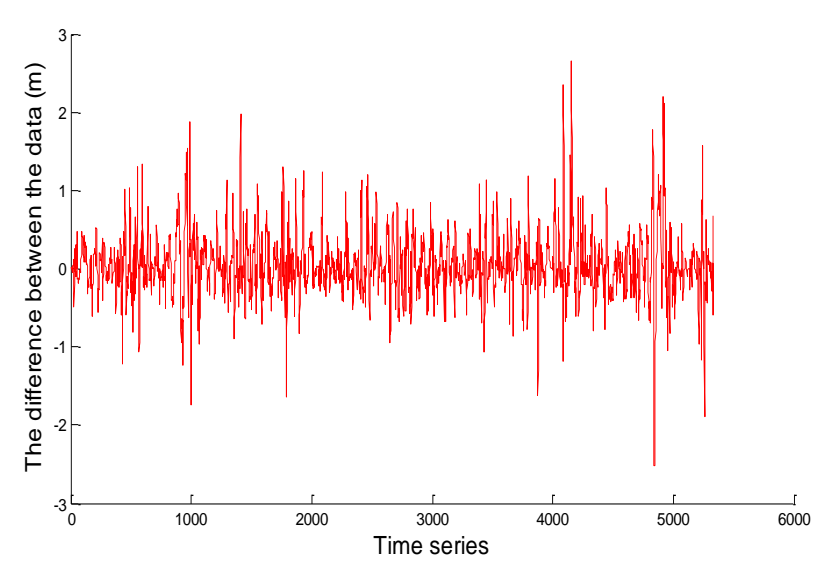

Fig. 6 the difference between the results of the two fusion methods

\section{Conclusion}

1) The median estimate is more robust than the mean value in the estimation of measure error distance of the last 5 frames, it effectively avoids the influence of outliers.

2) It is proved in this paper that the accuracy of multi-sensor data fusion is better than that of any sensor. On this basis, the multi-sensor data fusion method by combining nearest neighbor method and fuzzy inference is proposed. Since the nearest neighbor method is used to correct the data, this method is better than the weighted average fusion method based on fuzzy inference. The method proposed in this paper theoretically proves that the accuracy of data fusion will be higher rather than worse as the number of sensors increases, and it will have certain theoretical significance.

\section{References}

[1] Chui Bo.Key Technologies Research on Multi-sensor Data Fusion for Traget Tracking [D]. Southwest Jiaotong University, 2012.09.

[2] Huang Youpeng. Research on Serveral Key Techniques of Multi-sensor Multi-Target Track correlation and Data Combination [D]. Haebin Engineering University, 2009.06.

[3] Qian Huanming, Chen Liang, Man Guojing, et al. Adaptive tracking algorithm of maneuvering targets based on current statistical model [J]. Syestem Engineering and Electronics, 2011, 10(10): 2154-2158.

[4] Ren Shaowei, Wang Rui, Zhang Pingding. An Adaptive Algorithm of Maneuvering Targets Tracking Based on Maneuvering Frequency [J]. Journal of Air Force Engineering University (Natural Science Edition), 2004, 5(1): 32-35.

[5] Xia Peilun, Wen Hong. Target tracking based on fuzzy in ference [J]. Electronics Optics \& Contral, 2003, 10(1): 23-27.

[6] Li Qiuhua, Li Jicheng, Shen Zhenkang. Target tracking in the Dual Band IR Imageing Syestem Using Adeaptive weighting fusion Based on Fuzzy Inference [J]. Journal of Electronics \& information Technology, 2005, 27(12): 1922 -1926.

[7] Liu Xinwang, Huang Wei. Fuzzy inf erence based optimization method for multiobjective decision making [J]. Journal of Management Sciences in China, 2001, 12(4): 71-76.

[8] Lei Yang, Lei Jieying, et al. Techniques for target recognition based on adaptive intuitionistic fuzzy inference [J]. Syestem Engineering and Electronics, 2010, 7(7): 1471-1475. 